\title{
NASA Space Network Project Operations Management: Past, Present and Future for the Tracking and Data Relay Satellite Constellation
}

\author{
Ted Sobchak ${ }^{1}$, \\ Goddard Space Flight Center, Greenbelt, MD, 20771 \\ Donald W. Shinners ${ }^{2}$ \\ Goddard Space Flight Center, White Sands Complex, Las Cruces, NM, 88012 \\ Harry Shaw ${ }^{3}$, \\ Goddard Space Flight Center, Greenbelt, MD, 20771
}

\begin{abstract}
The NASA Space Network (SN) Operations began with the launch of the first Tracking and Data Relay Satellite (TDRS-1) on April 4, 1983 with on-orbit operations conducted from the White Sands Ground Terminal (WSGT) in Las Cruces, New Mexico. Over the past 35 years, the SN has evolved to its current configuration comprised of four strategically located satellite ground stations and a constellation of ten geosynchronous Tracking and Data Relay Satellites that provide customer support 24 hours a day, 365 days per year, at an unprecedented 99.9\% proficiency. The Space Network provides total global Communications, Data Relay, and Tracking services for Low Earth Orbiting (LEO) satellites, Human Space Flight, Expendable Launch Vehicles (ELV) and Scientific missions. This paper will address the Operations Management of the NASA Space Network and the complexities associated with this responsibility, as well as significant mission support highlights.
\end{abstract}

\section{Introduction}

$\mathrm{T}$ he Space Network is designed as a highly automated user-driven system that has evolved over time to provide extremely reliable support for customer spacecraft tracking and data acquisition. Each TDRS can simultaneously provide up to two S-Band Single Access (SSA) Forward (SSAF) and Return (SSAR) services, two Ku-Band Single Access (KSA) Forward (KSAF) and Return (KSAR) services, one Multiple Access (MA) Forward service, MA Return services, one-way Doppler and two-way Doppler and range services. Six of the TDRS (TDRS-8, 9, 10, 11, 12, and 13) can provide Ku-band or Ka-Band services. With TDRS spacecraft located in three geosynchronous regions around the earth (the Atlantic Ocean region, the Pacific Ocean region, and the Indian Ocean region) as depicted in Figure 1, customer spacecraft can attain total global support.

The Space Network, under the direction of the Goddard Space Flight Center (GSFC) Exploration and Space Communications (ESC) Projects Division is managed by the Space Network Project Office (Code 452). The SN Operations Center, located at the White Sands Complex (WSC) in Las Cruces, New Mexico, includes two satellite ground stations, the Second TDRS Ground Terminal (STGT) that encompasses Space-to-Ground Link Terminals (SGLT) 1, 2, and 3 and the White Sands Ground Terminal (WSGT) that is comprised of SGLT 4 and 5. SGLTs are dynamically configured to support a single TDRS at a time. SGLT-TDRS pairing is flexible based on spacecraft operational requirements and system availability. The WSGT and STGT ground stations maintain the Tracking, Telemetry, and Command (TT\&C) Space-to-Ground Link (SGL) with TDRS-3, 5, 6, 10, 11, 12 and 13 spacecraft.

\footnotetext{
${ }^{1}$ Project Manager, Space Network Project, Code 452, Greenbelt, MD 20771.

${ }^{2}$ Station Director, SN Project, Code 452, White Sands Complex, 12600 NASA Rd., Las Cruces, NM 88012.

${ }^{3}$ Staff Engineer, Telecommunications and Network Technologies Branch, Code 566, Greenbelt, MD 20771. 
The Guam Remote Ground Terminal (GRGT) was commissioned in July 1998 with the relocation of SGLT-6 from WSGT to Guam. This was necessary to allow closure of the zone of exclusion (ZOE), an area over the Indian Ocean where any supporting TDRS's could not communicate directly with the WSC and customers could not be supported by the SN. In the early 2000's, the Space Network Expansion (SNE) project installed an additional Space-to-Ground Link Terminal at the GRGT (SGLT-7). Currently, the Guam Remote Station (GRS) is comprised of SGLT-6 and 7 and maintains the TT\&C Space-to-Ground Link with TDRS-7 and 8.

In September 2015, the SN established in Welcome, Maryland, the Blossom Point Remote Station (BPRS). An eighth Space-to-Ground Link Terminal (SGLT-8) was installed that maintains the TT\&C link to TDRS-9. In addition to the aforementioned ground stations, two ancillary remote ground stations exist for emergency spacecraft recovery situations. The Extended TDRS Ground Terminal (ETGT) which is co-located at the WSGT and provides contingency S-band TT\&C support for the WSC, and the Australian TDRS Facility (ATF) located in Yatharagga, Western Australia that provides contingency S-band TT\&C support for the GRS.

The SN typically provides between 450 to 600 hours of customer support every day. Customers supported include: International Space Station (ISS), Hubble Space Telescope (HST), AIM, AQUA, AURA, GPM, LSAT-7, LSAT-8, MMS-1, 2, 3 \& 4, McMurdo TDRS Relay Station-2 (MTRS-2), NEOWISE, NUSTAR, SORCE, South Pole TDRS Relay-2 (SPTR-2), TERRA, H-II Transfer Vehicle (HTV), ATLAS Launch Vehicles, DELTA Launch Vehicles, SpaceX/Dragon, and Orbital ATK Cygnus. The Space Network provides around the clock support to the International Space Station to include all communications, all critical support, and all Extravehicular Activities (EVA's). This same level of support was provided to the Space Transportation System (STS) from 1994 until the final mission of STS135 in 2011.

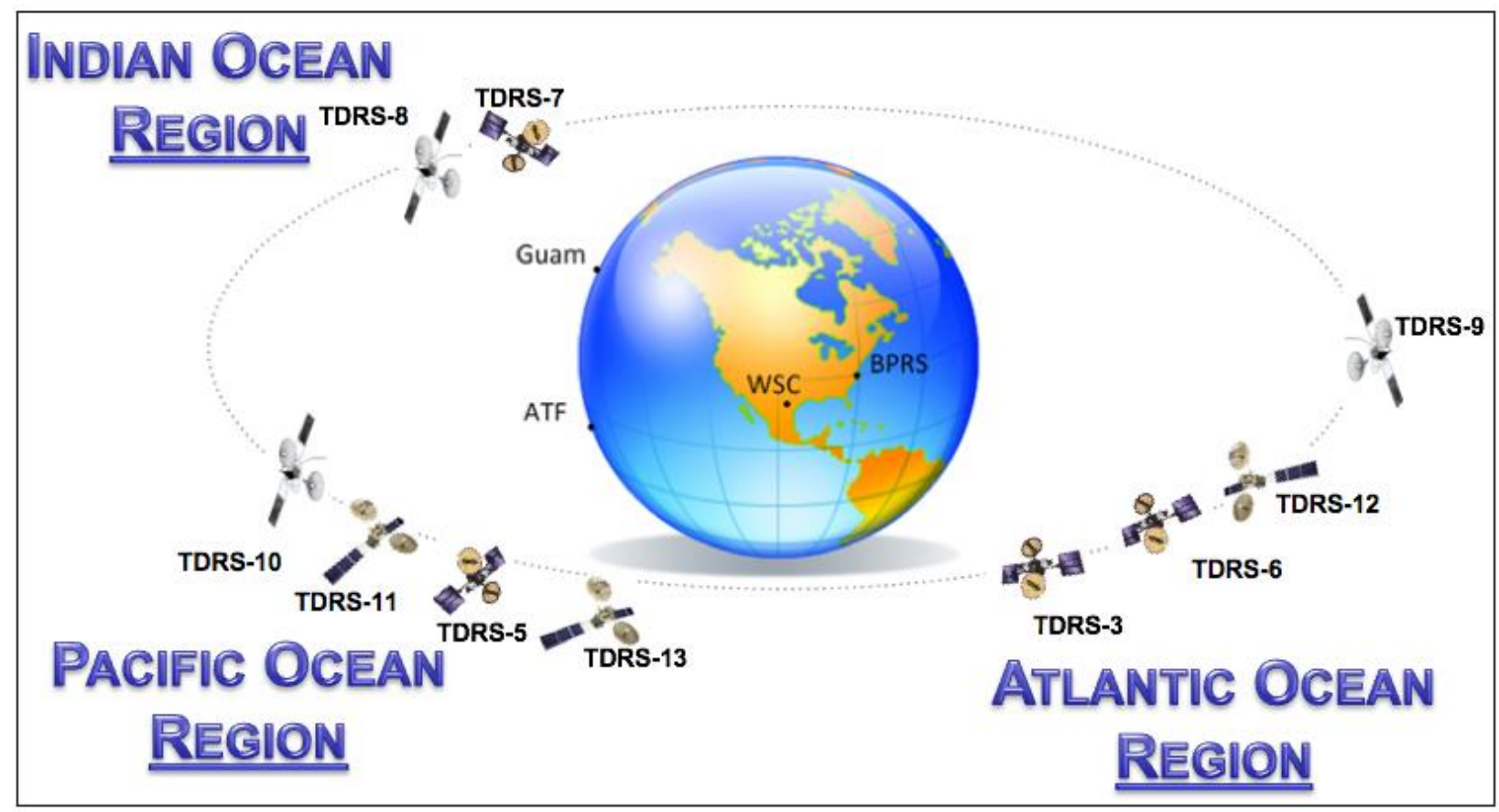

Figure 1. Three SN TDRS Nodes

While the current constellation of TDRS satellites and the Ground Segment have served reliably for decades, the future of the constellation will evolve to meet the changes of the $21^{\text {st }}$ Century space communications environment. In this paper, we will discuss evolutionary plans to adapt to:

- Demand for higher data rates and greater coverage from the ISS to support high rate experiments.

- Emergence of CubeSats as a consumer of space communications resources.

- The need for extensive ground system modernization to adopt a digital processing communications and networking environment with more flexibility to instantiate commercial off the shelf telecommunications technology.

American Institute of Aeronautics and Astronautics 


\section{Background}

$\mathrm{T}$ he Space Network initiated a new, cost-effective telecommunications network for NASA's space operations. The SN was established for two fundamental reasons: 1 . NASA projects greatly increased demand to handle data and tracking information from spacecraft and the Shuttle missions, and (2) the SN reduced the costs associated with servicing this increased demand. The Space Network allowed NASA to close, or transfer to other agencies, several ground stations around the globe while opening only one new ground station in New Mexico. The SN eliminated the need to establish and maintain a large number of ground stations required to supply equivalent services. NASA decided on the location of the ground terminal using very specific criteria. Foremost was the ground station's view of the satellites, and the location had to be close enough to the equator to view both the east and west skies. Weather was another important factor, New Mexico has, on average, almost 350 days of sunshine per year, with a very low precipitation level.

The original implementation of NASA's Space Network in the 1970's and 80's, was handled through a lease arrangement with a privately-held company known as Space Communications Company (Spacecom). Spacecom was a jointly owned subsidiary of Western Union Communications, Inc., Fairchild Industries, and Continental Telephone Company. The NASA lease covered a period of ten years during which time service would be provided by Spacecom in accordance with the provisions of their contract. The system leased by NASA required Spacecom to purchase both the spacecraft (TDRS) and launch services, as well as provide the Ground Terminal located at White Sands, NM. The principal elements of the TDRSS included the spacecraft, the ground terminal, and TDRSS services, as well as the launch system and operational activities.

The White Sands Ground Terminal contained all the necessary ground control equipment and associated systems and services that connected the NASA customer traffic interface and the orbiting Tracking and Data Relay Satellites. WSGT also provided the supporting customer link data-relay functions and monitored and controlled the Tracking and Data Relay Satellites. The WSGT also contained the NASA-owned portion of the user traffic interface, the NASA Ground Terminal (NGT). The NGT provided the interface for communications between the WSGT ground system and the remote customer systems and the NASA facilities. The NGT provided the multilink path of communications between the spacecraft and customer's project operations control center.

The Space Network was originally designed to have one ground terminal in White Sands, New Mexico, communicating with TDRS spacecraft at 41 degrees and 171 degrees West Longitude, with one spare TDRS on orbit. From there, WSGT would then forward the customer mission data received to each mission's science data processing center. With this system, missions such as the Space Shuttle, LandSat, and Hubble Space Telescope (HST), had access to low rate health and welfare data as well as high science data rate coverage (up to $300 \mathrm{Mbps}$ ) for up to $85 \%$ of their orbits. The original TDRS design would also provide commercial services, such as C-band transponders, for fixed earth station users. As originally conceived, the System was commercially owned and operated, and NASA would buy data services from the commercial owners. Thus, the original six (TDRS 1-6) first generation spacecraft were built with communication hardware to support the commercial customer set. TDRS-3 was launched in 1988, TDRS4 in 1989, TDRS-5 in 1991, and TDRS-6 in 1993.

Due to a variety of reasons, it was decided that it was in the best interest of the NASA community for NASA to own, manage, and operate the System. NASA purchased the TDRS spacecraft and ground terminal (WSGT) from the commercial owners in the early 1990s. During this time, another satellite ground station was being built three miles north of the WSGT facility. This new ground terminal was called the Second TDRS Ground Terminal (STGT) using state-of-the-art communications and computer equipment and systems designated as Space-to-Ground Link Terminals (SGLTs) developed by General Electric (GE) in Valley Forge, Pennsylvania. The WSGT and STGT are geographically separated and completely independent of one another, while retaining a fiber-optic link to transfer data between the two sites.

In December of 1994, the STGT was commissioned operational and became the primary Space Network Operations Control Center consisting of three Space-to-Ground Link Terminals (SGLT-1, 2, 3) and the main TDRS Operations Control Center (TOCC). In January 1995, the WSGT was decommissioned to begin removal of the original ground terminal communication systems, excluding the antennas, and install three more Space-to-Ground Link Terminals (SGLT-4, 5, 6) that became operational in 1996. Additionally, TDRS-7 (replacement spacecraft for TDRS2 that was destroyed in the 1986 Challenger explosion) was launched in July 1995, became operational at the end of

American Institute of Aeronautics and Astronautics 
the year. Even with the inclusion of the six new Space-to-Ground Link Terminals and the addition of TDRS-7, bringing the constellation up to six satellites, the SN still was limited to providing $85 \%$ orbital coverage for the majority of our customers. The remaining 15\% that was uncovered is designated as the TDRS Zone of Exclusion.

After a proof of concept design, it was determined that a SGLT could be placed on the island of Guam and relocating a TDRS over the Indian Ocean would provide SN customers with $100 \%$ global coverage. With the collaboration of the U.S. Navy, the SN proceeded to remove SGLT-6 from the WSGT, ship and reinstall it in a vacant building on the Naval Computer and Telecommunications Station (NCTS) in Guam. An 11-meter S/K-band Main Mission antenna and an End-to-End test antenna (4.5-meter) was relocated as well. TDRS-3 was relocated from a Pacific Ocean orbital location to an Indian Ocean orbital location to support the new SGLT-6. A Space Network Operational Readiness Review (ORR) was conducted and the Guam Remote Ground Terminal (GRGT) became fully operational in July 1998 as an extension of WSGT and an integral part of the NASA Space Network closing the Zone of Exclusion (ZOE).

Concurrently with the GRGT effort, NASA contracted with Boeing to build the second-generation TDRS spacecraft TDRS-H, I, and J. TDRS-H was launched in 2000 and TDRS-I and TDRS-J were launched in 2002. The second-generation spacecraft has the same capabilities as the first generation TDRS (1-7) but added Ka-band. The primary difference between the $2^{\text {nd }}$ generation TDRS and the $1^{\text {st }}$ generation TDRS is the MA beamforming being performed on-board the $2^{\text {nd }}$ generation spacecraft. Block diagrams of the $1^{\text {st }}$ generation and $2^{\text {nd }} / 3^{\text {rd }}$ generation spacecraft are shown in figure 2. There are thousands of permutations of coding, modulation, and data rates supported within the Space Network. However, there are four important general points for users to understand:

- Forward services refer to the link from the customer terrestrial location, to WSC, to TDRS, to the user spacecraft.

- Return services refer to the link from the user spacecraft, to TDRS, to WSC, to the customer terrestrial location.

- The space-to-space link between the user and TDRS can be at S-band, Ku-band or Ka-band

- The space-to-ground link carrying user data between TDRS and WSC is at Ku-band.

Table 1 summarizes provides a high level summary of the available user capabilities

Table 1. TDRS forward and return user capabilities

\begin{tabular}{|l|l|l|l|l|}
\hline \multicolumn{5}{|c|}{ Forward } \\
\hline & $\begin{array}{l}\text { Multiple Access } \\
\text { S-band }\end{array}$ & $\begin{array}{l}\text { Single Access } \\
\text { S-band }\end{array}$ & $\begin{array}{l}\text { Single Access Ku- } \\
\text { band }\end{array}$ & Single Access Ka-band \\
\hline $\begin{array}{l}\text { Number of user spacecraft per } \\
\text { TDRS }\end{array}$ & $1 *$ & 2 & 2 & 2 \\
\hline 3 dB RF Channel BW, MHZ & 6 & 20 & 50 & 50 \\
\hline Users Guide Max Data Rate & $300 \mathrm{kbps}$ & 7 Mbps & $25 \mathrm{Mbps}$ & $25 \mathrm{Mbps}$ \\
\hline \multicolumn{5}{|c|}{ Return } \\
\hline
\end{tabular}

- $\quad$ *Direct Spread Spectrum, details available upon request

- $\quad$ **The TDRS channel is capable of higher data rates with user supplied ground equipment. It has been characterized to up 3Gsps. 


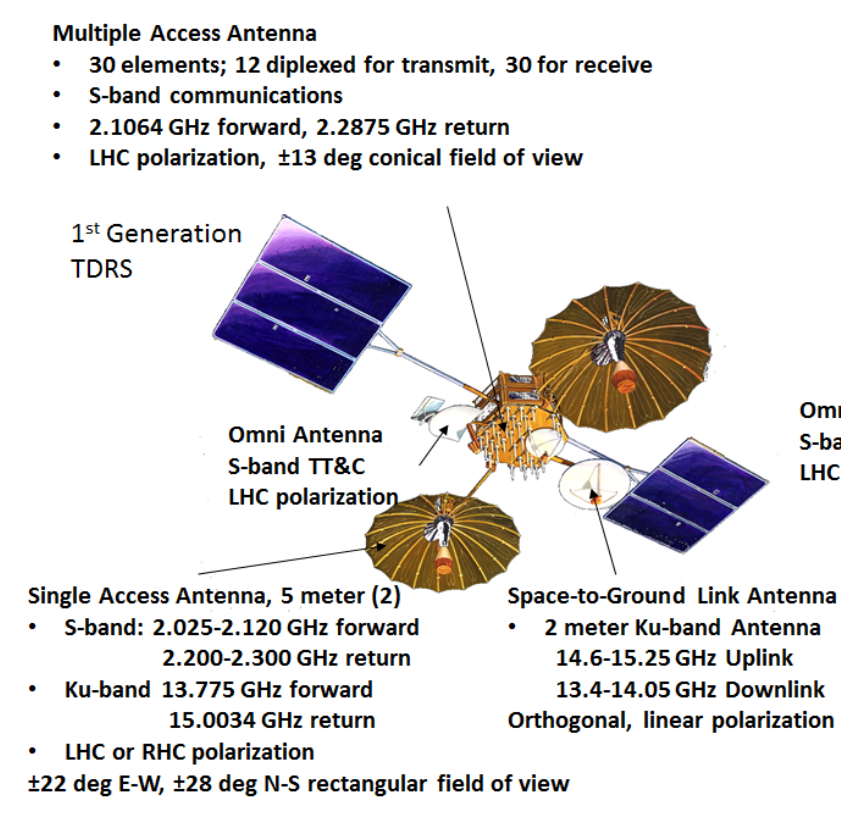

Single Access Antenna, 5 meter (2)

- S-band: $2.025-2.120 \mathrm{GHz}$ forward 2.200-2.300 GHz return

- Ku-band: $13.775 \mathrm{GHz}$ forward $15.0034 \mathrm{GHz}$ return

- Ka-band: $22.55-23.66 \mathrm{GHz}$ forward 25.25-27.50 GHz return

- LHC or RHC polarization

\pm 22 deg $E-W, \pm 28$ deg $N-S$ rectangular field of view \pm 30.5 deg $\mathrm{N}-\mathrm{X}$ elliptical extended field of view

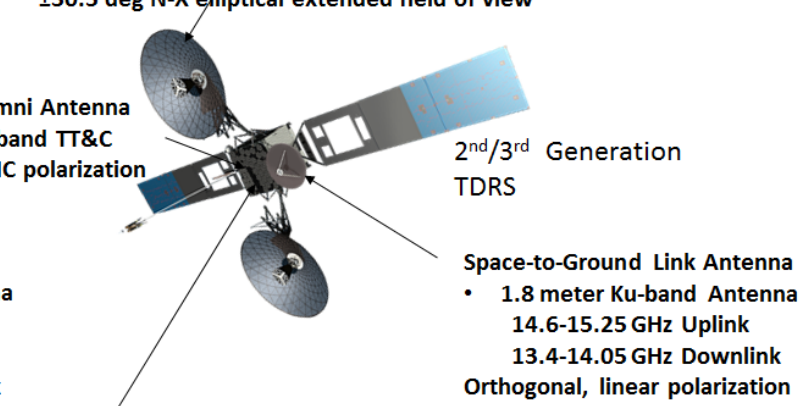

Multiple Access Antenna

- 32 elements; 15 diplexed for transmit, 32 for receive

- S-band communications

- $\quad 2.1064 \mathrm{GHz}$ forward, $2.2875 \mathrm{GHz}$ return

- LHC polarization, \pm 13 deg conical field of view

Figure 2. $1^{\text {st }}, 2^{\text {nd }}$, and $3^{\text {rd }}$ generation TDRS spacecraft architecture

In 2002, the SN expanded the TDRS Multiple Access (MA) return (spacecraft-to-ground) capabilities by developing the Demand Access System (DAS). DAS provides a low-data rate, low-cost return telemetry data service for spacecraft in earth orbit. It provides both dedicated, continuous $(24 \times 7)$ links for receiving telemetry data and singleevent "on-demand" data service enabling missions to expeditiously re-point instruments for observation of cosmic and earth-based events or for receiving alert messages. DAS is completely automated and requires very little operator intervention.

Additional ground terminal assets were needed to expand the network to meet an increasing SN customer demand for services, balance the load, and maximize the use of the TDRS spacecraft. In 2008, the Space Network Expansion (SNE) Project added ground terminal assets with the Space Network Expansion-West system (SNE-West) that added an additional Space-to-Ground Link Terminal (SGLT-7) and two additional S/K-band 20-meter Main Mission antennas at the GRGT. The addition of SGLT-7 resulted in the new Guam Remote Station (GRS).

On October 21, 2009, the SN decommissioned TDRS-1 and raised it from geosynchronous orbit to supersynchronous altitude to limit orbital debris, expelled all residual propellant from the propellant tanks, performed final passivation, turned off telemetry and terminated commanding. TDRS-4 was officially decommissioned on December 9, 2011 due to battery failures. TDRS-4 was raised to super-synchronous altitude, expelled any remaining propellant, performed final passivation and turned off telemetry and terminated commanding. These two efforts are noteworthy given the fact that neither spacecraft was designed with a concept of operations for an end of mission plan.

In 2014, the SN collaborated with the U.S. Navy at the Navy Research Laboratory (NRL) in Blossom Point, Maryland. The SNE project installed ground terminal assets with the Space Network Expansion-East system (SNEEast) a Space-to-Ground Link Terminal (SGLT-8) with two S/K-band 20-meter Main Mission antennas. A Space Network Operational Readiness Review (ORR) was conducted and in September, 2015, the Blossom Point Remote Station (BPRS) became an operational asset of the NASA Space Network.

The Boeing Company was contracted to build and launch the Third Generation TDRS (K, L, and M) spacecraft. TDRS-K was launched in 2013, TDRS-L in 2014, and TDRS-M on August 17, 2017. TDRS-K, L, and M have the same capabilities as the first and second generation TDRS with S-band, Ku-band, and Ka-band Single Access and Sband Multiple Access but the MA beamforming is ground based at the ground terminal. At this time, the SN consists of six Satellite Ground Terminals and ten Tracking and Data Relay Satellites. 


\section{Managing Change in the Space Network}

Tn 1999, the SN was challenged to reduce costs and increase efficiencies under the Consolidated Space Operations Contract (CSOC). At that time, the SN was operating the separate control centers at STGT and WSGT. It was decided to consolidate both control centers at the STGT and create one main TDRS Operations Control Center (TOCC). Additionally, a decision was made to relocate the functions of the Network Control Center (NCC) from the Goddard Spaceflight Center to the White Sands Complex and establish the Data Services Management Center (DSMC). The NCC provided the management functions that schedules, controls and monitors the Spaceflight Tracking and Data Network (STDN) which consists of the Space Network, the Ground Network (GN), and all support facilities necessary to provide telemetry, tracking, and command services to customers. The DSMC replaced many of the functions previously performed by the NCC, centralized the scheduling, service accounting, and monitoring and control of NASA's GN and SN resources. Additionally, some of the functions performed at the NCC were merged with the newly created DSMC positions including, the Operations Supervisor (OS), Site Specialist (SS), and the Communications Services Controller (CSC). Figure 3 shows the interfacing of the WSC/DSMC to various NASA and government elements.

In addition to the creation of the DSMC, a Technical Operations and Analysis (TO\&A) group was established in the SN Operations department to perform the NCC Network Integration and Analysis (NIA), Mission Operations Support Area (MOSA), STDN Mission Manager (SMM), and Network Systems Integration and Analysis (NSIA) functions at the WSC. The DSMC scheduling element consolidated and centralized the SN and GN scheduling functions while decommissioning the Ground Network Scheduling System Replacement (GNSSR) at the NCC. The Network Control Center Data System (NCCDS) was also relocated from GSFC to the WSC. This system was the NCC's primary service planning tool providing service planning database, schedule messages, scheduling rules, service control, service assurance, service accounting and system operation. The creation of the DSMC provided savings in operations and sustaining costs by reducing duplication and overlap of position functions and by streamlining the process to deliver data services to the customer while maintaining quality and reliability

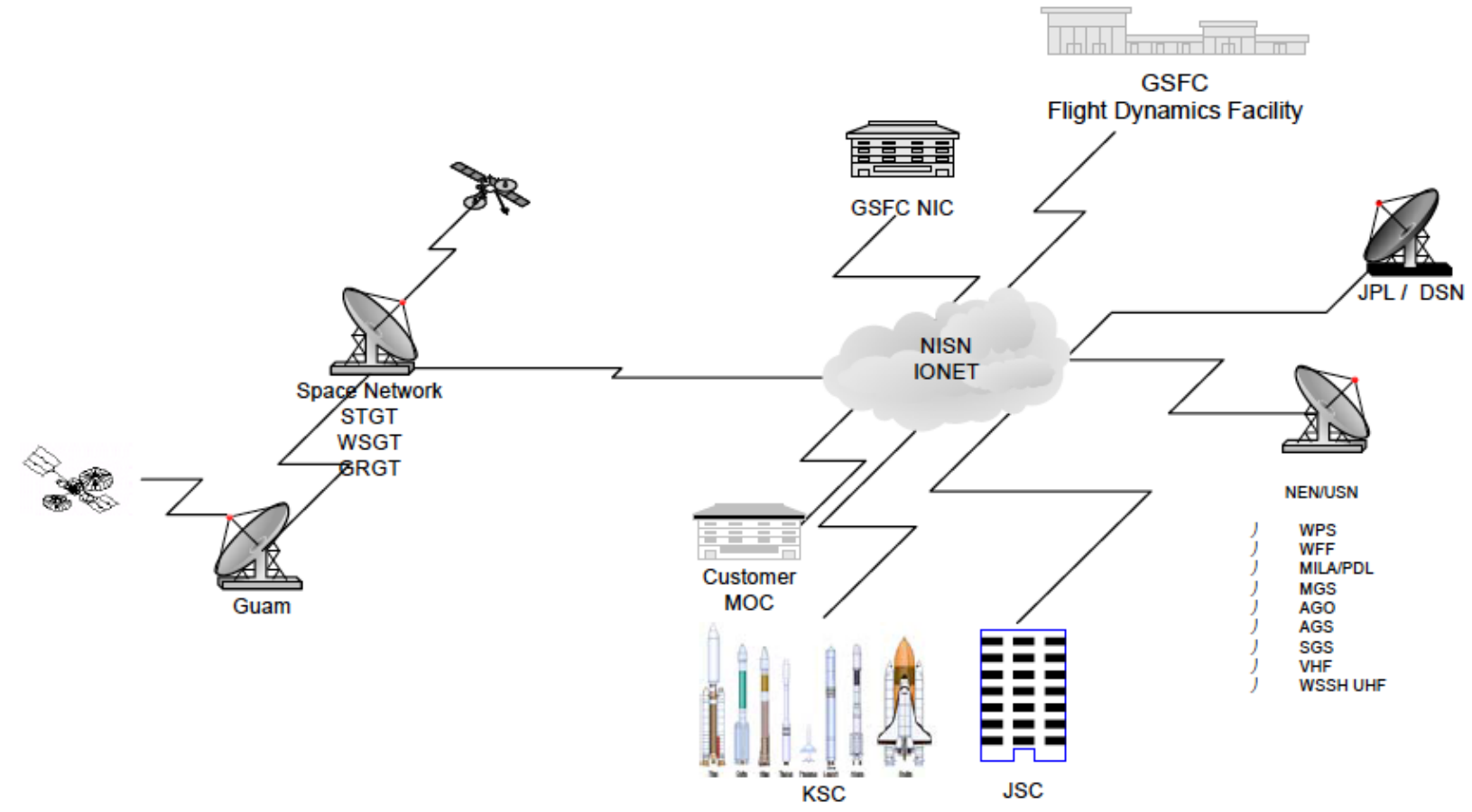

Figure 3. WSC/DSMC Interfaces

In 1984, the Space Network consisted of one satellite ground terminal, the WSGT, the NGT, and one TDRS with a total workforce of 280 personnel. Today, the SN has four satellite ground terminals, two auxiliary ground terminals, and ten TDRS with a workforce of 390 personnel. With the reduction in personnel and maximizing system efficiencies, the Space Network has been performing exceptionally well as depicted in figure 4.

American Institute of Aeronautics and Astronautics 


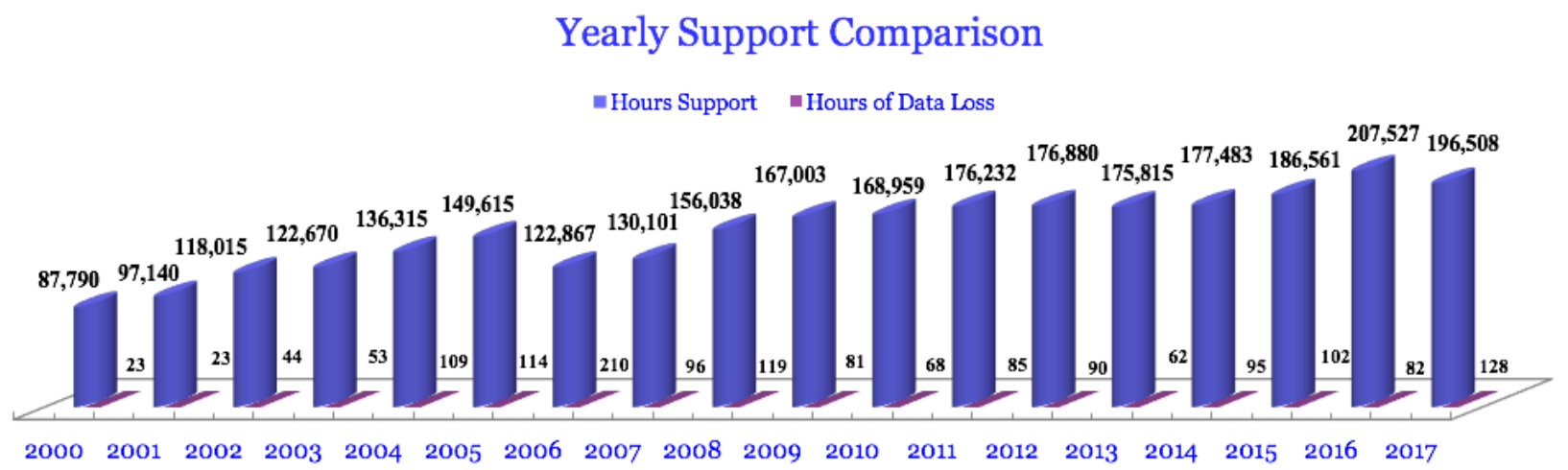

Figure 4. Space Network Operations Proficiency

\section{A. TDRS Digital Signal Distribution (TDSD)}

\section{Evolution of the Space Network}

$\mathrm{O}_{\mathrm{min}}^{\mathrm{nen}}$ ne of the most important upgrades to WSC was the introduction of TDSD. TDSD provides digital distribution of
TDRSS Return services. These services were implemented with WSC even though a digital architecture was not contemplated in the original ground network design. The main features are:

- Ku-band SGL downlinks are block-downconverted by the TDSD infrastructure and then digitized.

- Digitization occurs at a point close to SGLT antenna (after the LNA)

- $\quad$ RF to L-Band Downconverter $(1.8 \mathrm{GHz})$

- Digitized sampling at L-Band (1.8 Gsamples per second)

- Distribution from TDSD is packet-based using $10 \mathrm{~Gb}$ Ethernet

- Packetization is done using the Signal Data Distribution Standard (SDDS) format

- Users can subscribe via UDP/IP multicast to SDDS Ethernet packets belonging to different return services from Packet Switch Matrix (PSM)

- TDSD includes Multiple Access Return beamformers

- TDSD systems deployed at all three SN ground terminals support TDRSS return services:

- Customers can subscribe to digitized, packetized IF data services for MAR, SMAR, SSAR, KuSAR, and KaSAR.

- TDSDs at STGT and WSGT are linked through the inter-facility fiber, and allows for TDSD visibility of all WSC SGLTs from either STGT or WSGT.

The TDSD system was used to implement upgrades to Space Network ISS support. The context of TDSD within WSC is shown in figure 5 . 


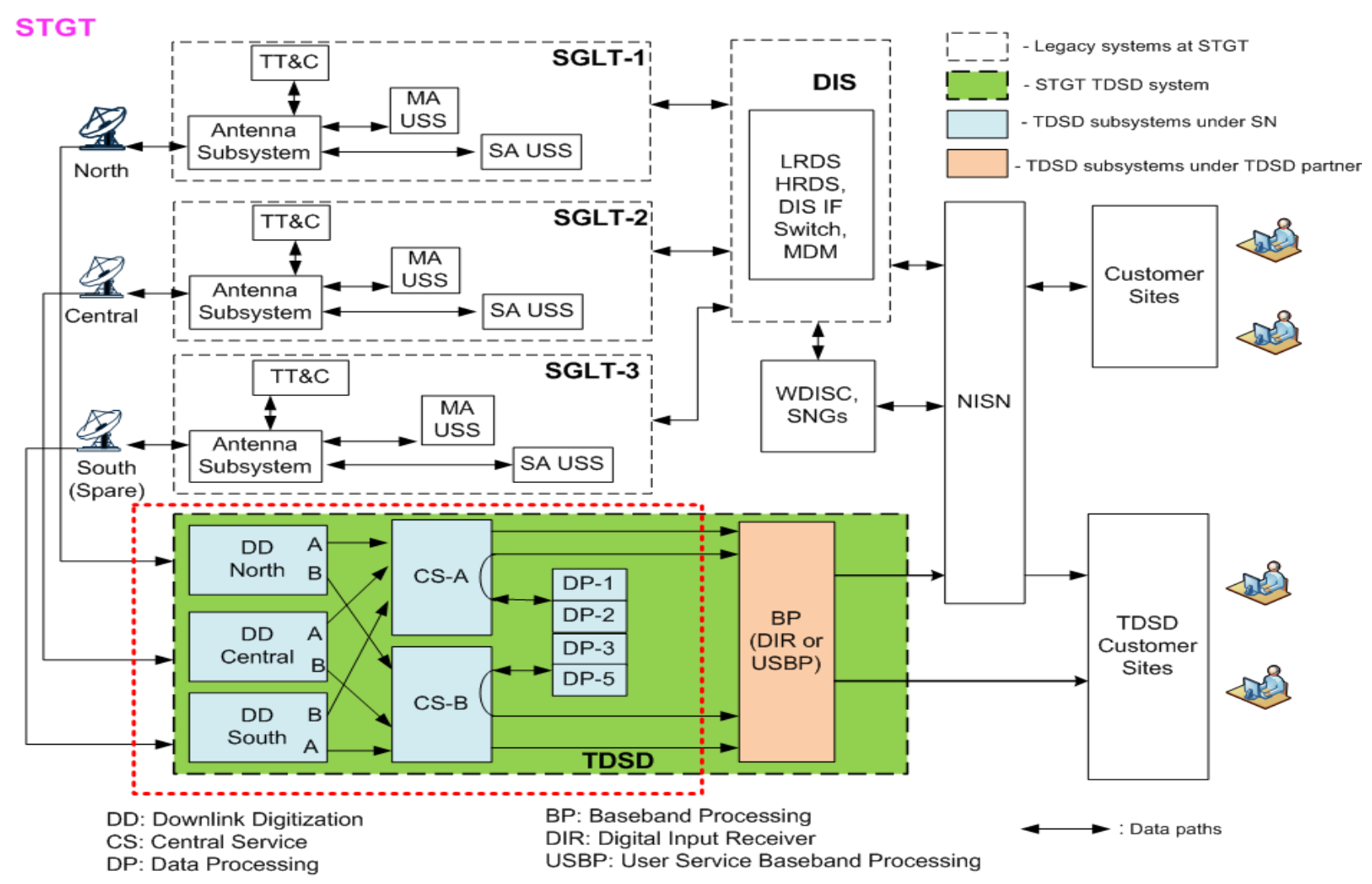

Figure 5. TDSD Ground Terminal Context

\section{B. Improvements to ISS support}

The most visible and important NASA TDRS support is to ISS. Voice, video and other high rate data comes from ISS through the TDRS 225MHz Ku-band Single Access Return Channel. Working in conjunction with Johnson Space Center and Boeing, the data rate from ISS has been increased incrementally from $150 \mathrm{Mbps}$ to $300 \mathrm{Msps}$ to $600 \mathrm{Msps}$ within the $225 \mathrm{MHz}$ channel. The forward error correction code was upgraded to LDPC Rate 7/8 and modulation upgraded to 8PSK. In a project titled EDISN (Enhanced Digital Infrastructure for the Space Network), new wideband digital modems were installed tied into the WSC digital infrastructure over TDSD. EDISN also included extended ISS coverage to include the ZOE over the Indian Ocean by upgrading the Guam station and extensive ground system modifications to the scheduling, command and control operations of the Space Network. A block diagram of EDISN is shown in figure 6.

American Institute of Aeronautics and Astronautics 


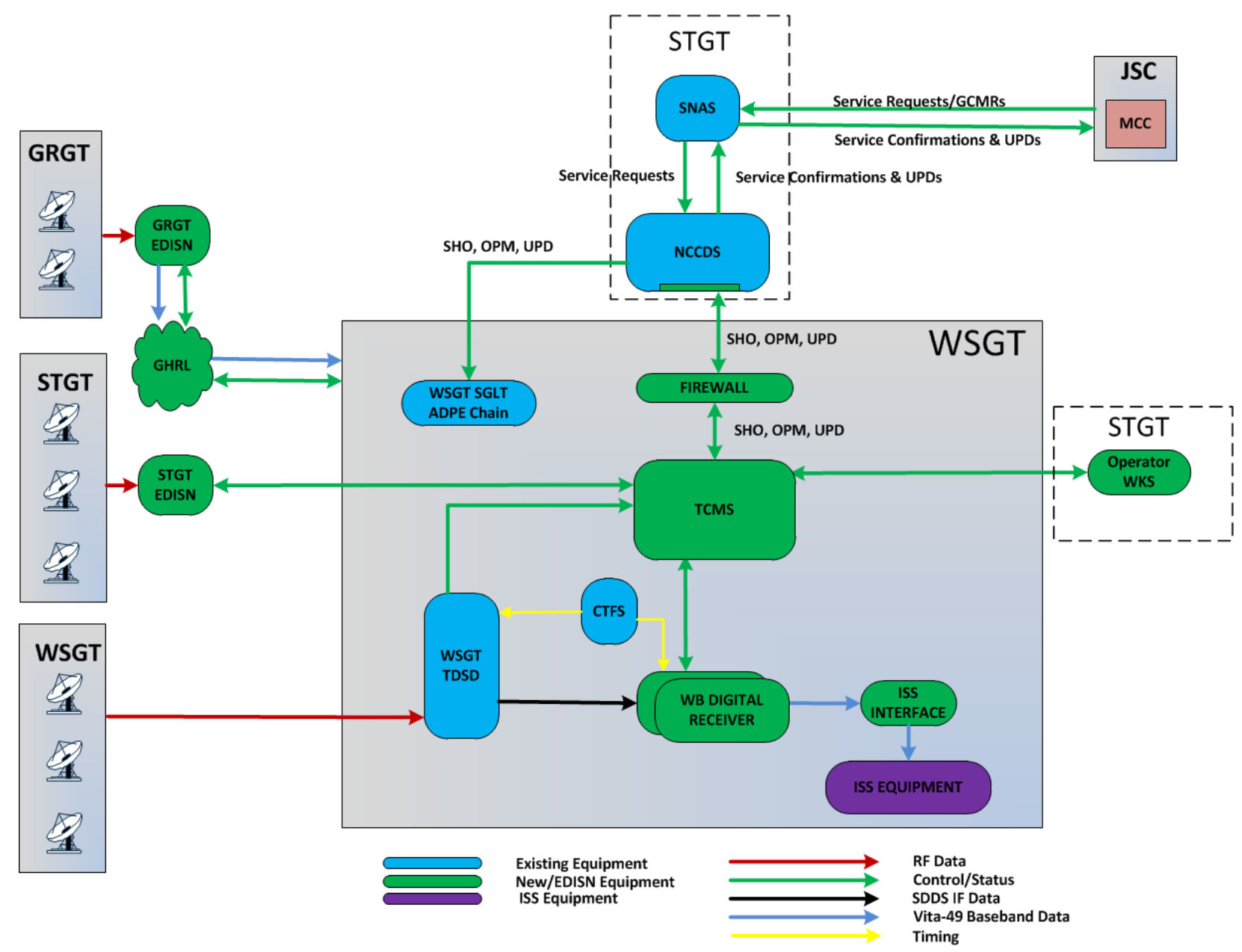

Figure 6. EDISN Architecture as the WSGT site. Schedules and service requests come into EDISN at WSGT via the Network Control Center Data System (NCCDS) which lies between the outward facing scheduler (SNAS) and the interior of WSGT network infrastructure. The heart of EDISN is the TCMS (TDSD Control and Monitoring System) SHO, OPM, UPD, and GCMRs are various message types required for system functionality. WSGT is connected to the other EDISN systems via WAN. Other acronyms: CTFS (Common Time and Frequency System), ADPE (Automated Data Processing Equipment), WKS (Workstation), GHRL (Guam High Rate Link), WB (Wideband)

\section{Swift mission and the Burst Alert Messages}

The Swift mission took advantage of $24 \times 7$ visibility of the SN relay satellites to institute a system of burst alert messages to permit rapid notification of gamma ray bursts throughout the Gamma Ray Burst Coordination Network. This permits the science community an opportunity for rapid observation of phenomena of interest. Shown below is a description of the process in figure 7 from the NASA/GSFC Swift mission website (https://swift.gsfc.nasa.gov/about_swift/mission_flow/gen_desc.html)

American Institute of Aeronautics and Astronautics 


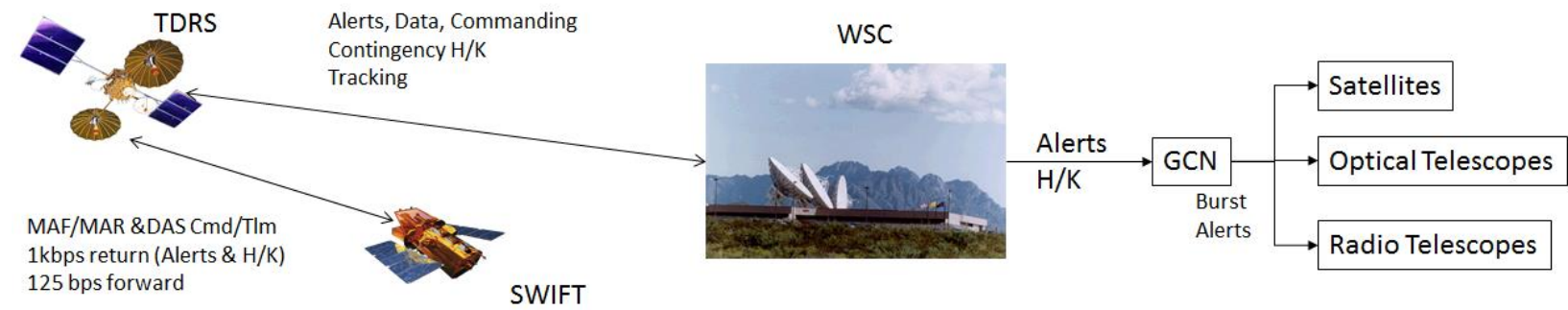

Figure 7. Swift Burst Alerts via TDRS and WSC. Swift is also able to utilize the Demand Access System which permits a simplified scheduling approach to utilize any TDRS currently in view with open capacity.

\section{TDRS and future support to CubeSat constellations}

CubeSats and SmallSats represent a rapidly growing segment of on-orbit spacecraft. Plans exist for constellations of thousands of spacecraft. This represents a potential congestion of the spectrum allocated for space-to-ground communications. TDRS has the potential to alleviate that spectrum congestion by use of the S-band direct spread spectrum multiple access return service. The Space Network can provide a range of services for CubeSats (including, but not limited to):

- $24 \times 7$ continuous communications coverage

- Post-separation communications

- Emergency communications

- Low rate continuous data

- Low latency data availability

- On-demand scheduling

- CubeSat constellation communication services

CubeSats and SmallSats would share the S-band MA forward and return frequency using unique pseudorandom codes. Figure 8 depicts one concept for supporting CubeSat constellations.

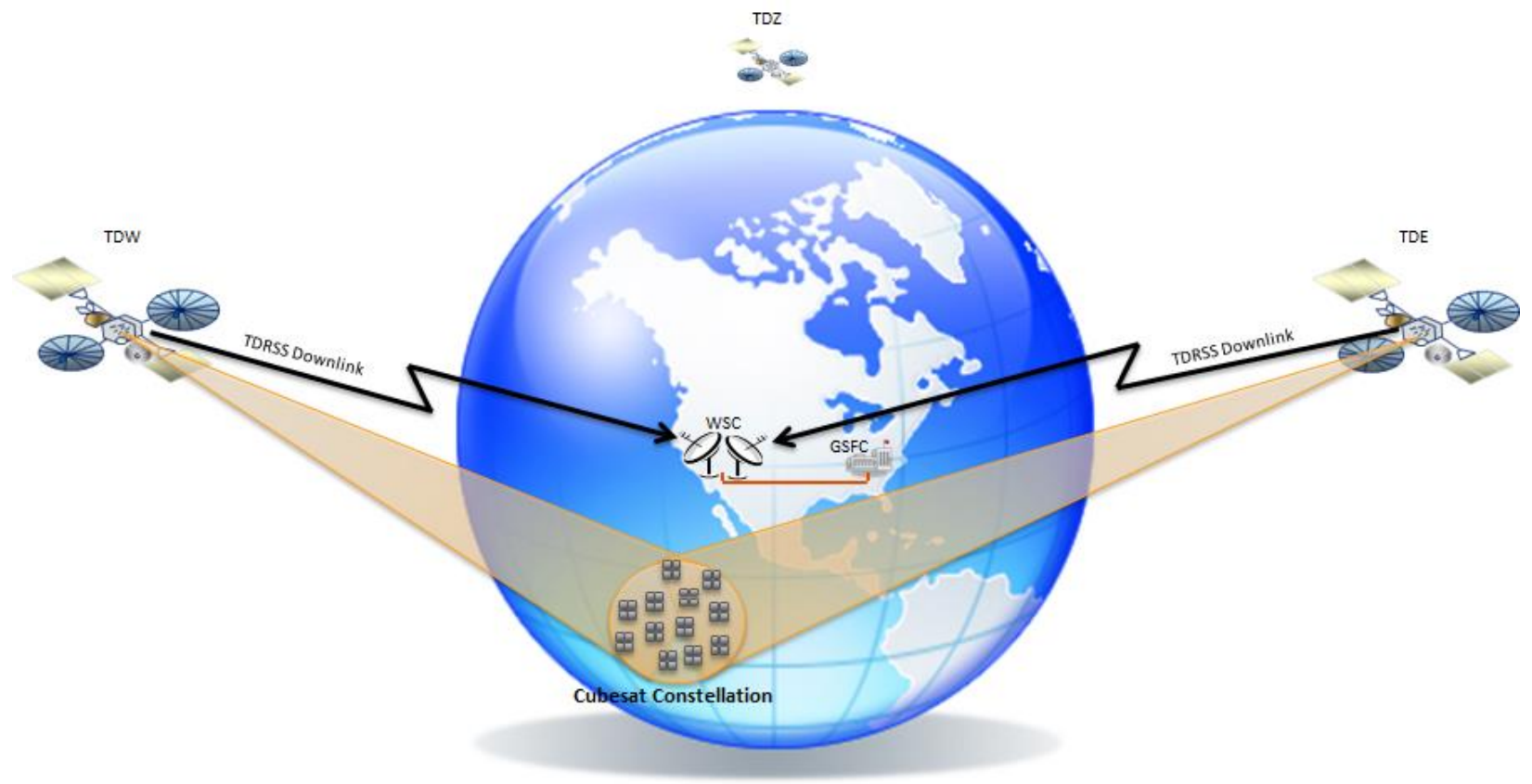

Figure 8. CubeSat constellations can communicate via TDRS using the Multiple Access Services.

American Institute of Aeronautics and Astronautics 
WSC is implementing a series of low-cost digital receivers and beamformers that are integrated with the WSC digital architecture to permit a large number of simultaneous supports of return services for CubeSats. This system is called Digital Demand Access Service (D-DAS). The architecture is described in figure 9.

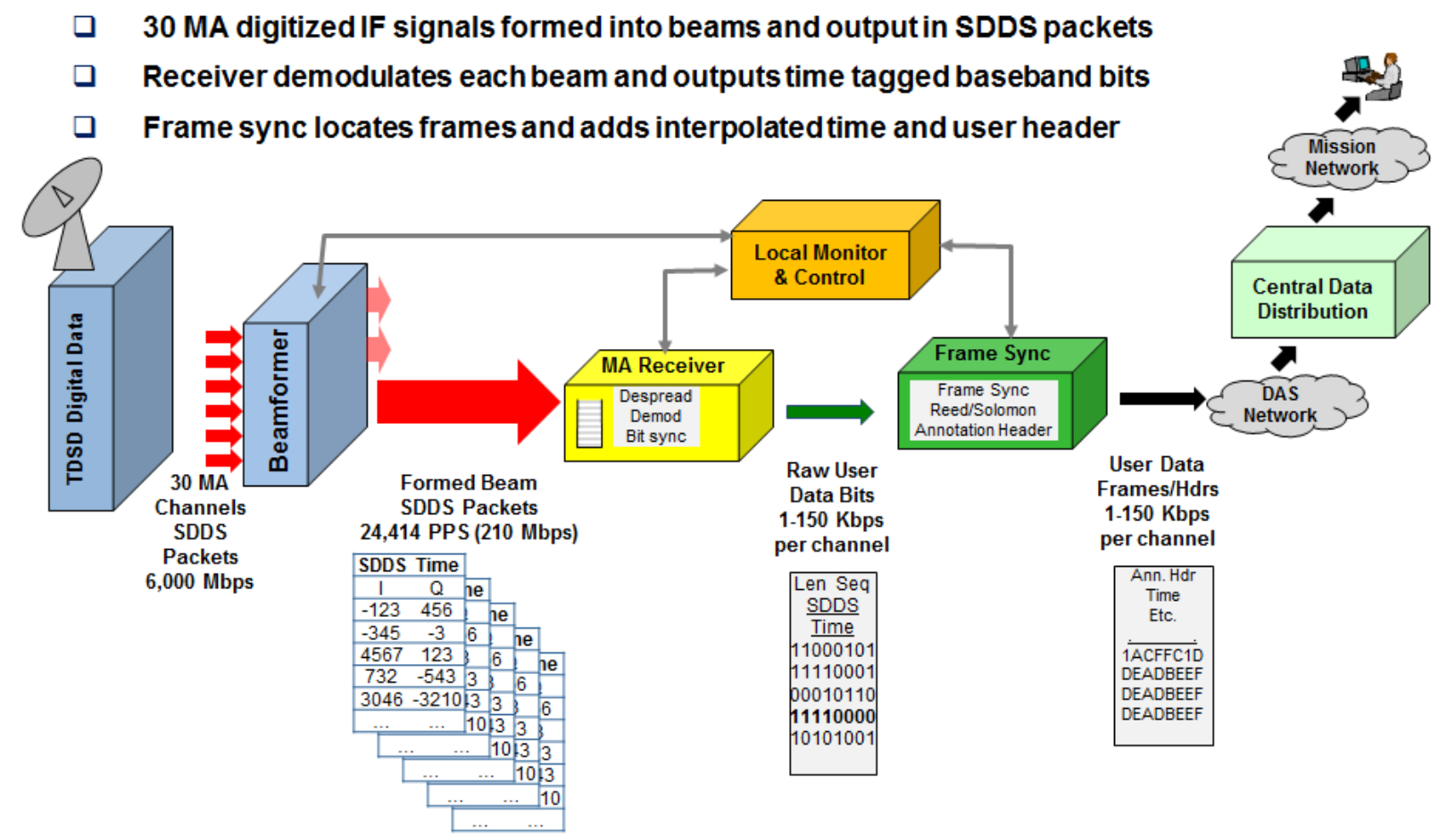

Figure 1. End-to-End D-DAS Signal Processing Architecture

GSFC is currently working on a variety of technologies to overcome some of the inherent problems with CubeSatTDRS communications including:

- Insufficient EIRP to close the link between LEO CubeSats and GEO TDRS. A number of technologies are under development including:

- High efficiency Solid State Power Amplifiers

- High gain antenna technologies

- Lack of a Commercial-Off-The-Shelf (COTS) spread spectrum radios for CubeSats.

- Investigation of a number of COTS and in-house radio development

\section{E. Plans for Future Operations - Optical Communications}

Operations of optical communications spacecraft and services are being performed at the White Sands Complex. The 2013 Lunar Laser Communications Demonstration (LLCD) and its ground segment (LLGT) was located at WSC. The Space Network and MIT/Lincoln Laboratory are operating the Laser Communications Relay Demonstration (LCRD) with a ground station installed at Haleakala, HI. LCRD is hosted aboard STPsat-6 launching in 2019. The STPsat-6 Mission Operations Center, LCRD Mission Operations Center (LMOC), and STPsat RF Ground Station are at WSC. Future plans for a Next Generation Earth Relay optical communications system will include optical ground stations at the WSC and geographically diverse locations. These stations will be capable of 100 Gbps optical space to ground links from optical relay satellites and user direct to earth optical communications links.

\section{Summary}

$\mathrm{T}$ The Space Network provides a diverse set of space communications services which has evolved over time. Three generations of TDRS relay satellites, evolution of the ground segment to incorporate a digital signal processing architecture and incorporation of operations of optical communications satellites and services has occurred over the

American Institute of Aeronautics and Astronautics 
last 40 years. The Space Network will continue to evolve and provide communications services in accordance with emerging needs trends and needs of NASA missions. 